\title{
The Inverse Periodic Spectral Theory of the Euler-Bernoulli Equation
}

\author{
Vassilis G. Papanicolaou \\ Communicated by Boris Mityagin, received October 13, 2004, and, in revised form, April 20, \\ 2005.
}

\begin{abstract}
The Floquet (direct spectral) theory of the periodic Euler-Bernoulli equation has been developed by the author in $[\mathbf{3 7}],[\mathbf{4 1}]$, and $[\mathbf{3 8}]$. A particular case of the inverse problem has been studied in [39]. Here we focus on the inverse periodic spectral problem. A key ingredient is an extended version of Abel's theorem for the existense of meromorphic functions on Riemann Surfaces. To avoid technicalities, we have assumed that the Floquet multiplier has finitely many branch points (in the Hill operator case this corresponds to the assumption that the spectrum has finitely many gaps).
\end{abstract}

\section{Contents}

1. Introduction 127

2. Review of the Floquet/Spectral Theory 129

3. The Riemann Surface $\bar{\Gamma} \quad 138$

4. A Brief Review of the Inverse Hill Theory 140

5. Extended Abel's Formula 142

6. Discussion on the Recovery of $a(x)$ and $\rho(x) \quad 145$

References 146

\section{Introduction}

The term "periodic Euler-Bernoulli equation" refers to the eigenvalue problem

$$
\left[a(x) u^{\prime \prime}(x)\right]^{\prime \prime}=\lambda \rho(x) u(x), \quad-\infty<x<\infty,
$$

1991 Mathematics Subject Classification. Primary 34A55, 34B05; Secondary 34B10, 34B30, 34L40, 74B05.

Key words and phrases. Euler-Bernoulli (or beam) operator, Euler-Bernoulli equation for the vibrating beam, Hill's operator, periodic coefficients, Floquet theory, spectrum, pseudospectrum, multipoint eigenvalue problem, inverse periodic spectral theory, Abel's theorem.

(C)2005 International Press 
where $a(x)$ and $\rho(x)$ are strictly positive and periodic with a common period $b$, satisfying the smoothness conditions $a \in C^{2}(\mathbb{R})$ and $\rho \in C(\mathbb{R})$ (we believe that these smoothness assumptions for $a$ and $\rho$ can be relaxed). Furthermore, without loss of generality, $a(x)$ and $\rho(x)$ are normalized so that

$$
\int_{0}^{b}\left[\frac{\rho(x)}{a(x)}\right]^{1 / 4} d x=b
$$

One advantage of this normalization is that the asymptotics of certain quantities, as $|\lambda| \rightarrow \infty$, become simpler, and this is the only reason that (2) is used in the present work.

The Floquet theory, i.e. the direct spectral theory, of (1) has been developed by the author in $[\mathbf{3 7}],[\mathbf{4 0}]$, and [38] (in chronological order). A special case of the inverse spectral problem has been studied in $[39]$. There are theoretical as well as practical reasons for studying (1). The spectral theory of (1) is richer (analytically as well as algebraically) than its second-order counterpart (namely the Hill's equation). All the main second-order properties continue to hold, while new interesting (we can say surprising) phenomena arise which are nonexistent in the second-order case. In fact, we believe that (1) is a good "representative" of higher-order periodic spectral problems, hence the full understanding of (1) will give insight to the general higher-order case.

On the practical side, we notice that a typical application of (1) is that it models the transverse vibrations of a thin straight beam with periodic characteristics (see, e.g., [46] or [20]) and elastic structures consisting of many thin elements arranged periodically are quite common [34].

In 1981 a third order periodic operator was studied by McKean in relation to the Boussinesq's flow (see $[\mathbf{2 7}]$ ). Recently there has been an increasing interest in higher order periodic eigenvalue problems (e.g. [6], [7]) and one expects that they will appear often in applied mathematics and mathematical physics as models of the physical world.

In the present work, in order to avoid obscuring complications, we develop the inverse periodic spectral theory of (1) under the assumptions (see Assumption I and II in Subsection 2.3) that the spectrum has finitely many nondegenerate gaps and the $\psi$-spectrum has finitely many nondegenerate $\psi$-gaps. The goal is a theory analogous to the theory of the Hill's operator $-(d / d x)^{2}+q(x)$, in the spirit of, say, $[\mathbf{1 4}]$ or $[\mathbf{1 3}]$ (see also $[\mathbf{1 0}],[\mathbf{1 5}],[\mathbf{1 7}],[\mathbf{3 0}],[\mathbf{3 1}],[\mathbf{4 8}]$ ).

In Section 2 we review some facts and notions from our previous works [37], $[41],[38]$, and [39], including the concept of the pseudospectrum, or $\psi$-spectrum. The section is broken into several subsections for the convenience of the reader. It contains certain observations never published before. The theorems presented in Section 2 are numbered by capital Latin letters to indicate that they are not new, but they have been proved in our previous works. Section 3 contains a description of the Riemann surface $\bar{\Gamma}$, namely the compactified surface of the Floquet multiplier $r(\lambda)$. Section 4 is a brief review of the (periodic) inverse spectral theory of the Hill's equation. We included this short section in order to get an understanding of what should be the main steps in developing the inverse theory of any periodic operator and, also, what are the problems one has to confront when one wants to go from the Hill case to the Euler-Bernoulli case. In Section 5 we show how to obtain a complete set of the evolution equations of the $\mu_{n}(x)$ 's, namely the poles of the normalized 
Floquet solutions, with the help of an extended version of Abel's theorem regarding meromorphic functions on Riemann surfaces. We discuss the Hill case first, as a "warm-up" example. We also derive some additional equations for the $\mu_{n}(x)$ 's and we finish with an example. The section is broken into four subsections. Finally, in Section 6 we discuss how one can, in principle, recover the coefficients $a(x)$ and $\rho(x)$ of (1) from the appropriate periodic spectral data.

One application of the theory presented in this paper is (see [28]) in solving nonlinear integrable systems of PDE's with periodic initial data, that can be written as Lax pairs involving the Euler-Bernoulli operator. In fact, recently J. Hoppe, A. Laptev, and J. Östensson (see [21]) have derived Lax pairs involving the general real self-adjoint fourth-order operator.

\section{Review of the Floquet/Spectral Theory}

We start by recalling some general facts about (1) and some results established in $[\mathbf{3 7}],[\mathbf{4 0}]$, and $[\mathbf{3 8}]$ (other references for Floquet or periodic spectral theory are, e.g., $[\mathbf{9}],[\mathbf{1 0}],[\mathbf{1 6}]$, Sec. XIII.7, $[\mathbf{1 9}],[\mathbf{2 2}],[\mathbf{2 4}],[\mathbf{2 5}],[\mathbf{4 2}])$.

The problem (1) is self-adjoint (with no boundary conditions at $\pm \infty$ ). The underlying operator $L$ (the "Euler-Bernoulli operator" or "beam operator") is given by

$$
L u=\rho^{-1}\left(a u^{\prime \prime}\right)^{\prime \prime} .
$$

The corresponding Hilbert space is the $\rho$-weighted space $L_{\rho}^{2}(\mathbb{R})$. Notice that $L$ is a product of two second order differential operators, namely

$$
L=L_{2} L_{1}, \quad \text { where } \quad L_{1} u=-a u^{\prime \prime}, \quad L_{2} u=-\rho^{-1} u^{\prime \prime} .
$$

If

$$
a(x) \rho(x) \equiv \text { constant, }
$$

the beam operator becomes the square of a second-order (i.e. Hill-type) operator.

2.1. Floquet Multipliers. For a given reference point $\xi \in \mathbb{R}$, let $u_{j}(x)=$ $u_{j}(x ; \xi ; \lambda), j=1,2,3,4$, be the fundamental solutions of (1) with respect to $\xi$, namely the solutions such that (primes denote derivatives with respect to the first variable, namely $x$, and $\delta_{j k}$ is the Kronecker delta)

$$
u_{j}^{(k-1)}(\xi ; \xi ; \lambda)=\delta_{j k}, \quad k=1,2, \quad a(\xi) u_{j}^{\prime \prime}(\xi ; \xi ; \lambda)=\delta_{j 3}, \quad\left[a u_{j}^{\prime \prime}\right]^{\prime}(\xi ; \xi ; \lambda)=\delta_{j 4} .
$$

Each $u_{j}(x ; \xi ; \lambda)$ is entire in $\lambda$ of order $1 / 4$. If $\xi=0$, we write

$$
u_{j}(x ; \lambda) \stackrel{\text { def }}{=} u_{j}(x ; 0 ; \lambda) .
$$

The corresponding Floquet matrix $T=T(\xi ; \lambda)$ is

$$
T=\left[\begin{array}{cccc}
u_{1}(\xi+b) & u_{2}(\xi+b) & u_{3}(\xi+b) & u_{4}(\xi+b) \\
u_{1}^{\prime}(\xi+b) & u_{2}^{\prime}(\xi+b) & u_{3}^{\prime}(\xi+b) & u_{4}^{\prime}(\xi+b) \\
a(\xi) u_{1}^{\prime \prime}(\xi+b) & a(\xi) u_{2}^{\prime \prime}(\xi+b) & a(\xi) u_{3}^{\prime \prime}(\xi+b) & a(\xi) u_{4}^{\prime \prime}(\xi+b) \\
{\left[a u_{1}^{\prime \prime}\right]^{\prime}(\xi+b)} & {\left[a u_{2}^{\prime \prime}\right]^{\prime}(\xi+b)} & {\left[a u_{3}^{\prime \prime}\right]^{\prime}(\xi+b)} & {\left[a u_{4}^{\prime \prime}\right]^{\prime}(\xi+b)}
\end{array}\right],
$$

where the dependence in $\xi$ and $\lambda$ is suppressed for typographical convenience. Notice that, if $\xi_{1}, \xi_{2} \in \mathbb{R}$, then $T\left(\xi_{1} ; \lambda\right)$ and $T\left(\xi_{2} ; \lambda\right)$ are similar matrices, hence the coefficients of the characteristic equation of $T(\xi ; \lambda)$ do not depend on $\xi$. 
In [37] it was shown that the eigenvalues $r_{1}, r_{2}, r_{3}, r_{4}$ of $T$ (called Floquet multipliers) appear in pairs of inverses, namely

$$
r_{1} r_{4}=r_{2} r_{3}=1
$$

(in fact this is true for any self-adjoint ordinary differential operator with real, periodic coefficients). Therefore the characteristic equation of $T$ has the (equivalent) forms

$$
r^{4}-A(\lambda) r^{3}+[B(\lambda)+2] r^{2}-A(\lambda) r+1=0,
$$

$$
\left(r+r^{-1}\right)^{2}-A(\lambda)\left(r+r^{-1}\right)+B(\lambda)=0 .
$$

It is more natural to view the $r_{j}$ 's as the branches of the analytic function $r(\lambda)$ "living" (defined) on a four-sheeted Riemann surface which we denote by $\Gamma$.

Remark 1. As we will see later, the asymptotics of $r(\lambda)$, as $|\lambda| \rightarrow \infty$ (formula (42)), implies that $r(\lambda)$ cannot be meromorphic at points at infinity, thus the surface $\Gamma$ is not compact since it misses the points at infinity. Furthermore, $r(\lambda)$ never vanishes on $\Gamma$, and because of that (and its asymptotics, as $|\lambda| \rightarrow \infty$ ) we can think of $r(\lambda)$ as playing the role of an "exponential function of $\Gamma$ ".

If we set

$$
r=e^{i k b}
$$

then the characteristic equation of $T$ becomes

$$
F(\lambda ; k) \stackrel{\text { def }}{=} B(\lambda)-2 A(\lambda) \cos (k b)+4 \cos ^{2}(k b)=0 .
$$

The function $F(\lambda ; k)$ is entire in $\lambda, k$ (it could be called the Akhiezer function). It is the analog of $\Delta(\lambda)-2 \cos (k b)$ of the Hill theory (where $\Delta(\lambda)$ is the Hill discriminant). Equation (7), which sometimes is called the dispersion relation, defines a transcendental variety, called the Bloch-Floquet variety. A detailed study of the zeros of $F(\lambda ; k)$, for any given $k \in \mathbb{C}$, can be found in [38].

The Floquet multipliers, being the roots of (5), satisfy

$$
r_{1}+r_{4}=\frac{A(\lambda)+\sqrt{E(\lambda)}}{2} \stackrel{\text { def }}{=} \Delta_{+}(\lambda), \quad r_{2}+r_{3}=\frac{A(\lambda)-\sqrt{E(\lambda)}}{2} \stackrel{\text { def }}{=} \Delta_{-}(\lambda),
$$

where $\sqrt{\cdot}$ is the principal branch of the square root function and

$$
E(\lambda) \stackrel{\text { def }}{=} A(\lambda)^{2}-4 B(\lambda) .
$$

Therefore

$$
\begin{array}{ll}
r_{1}=\frac{\Delta_{+}+\sqrt{\Delta_{+}{ }^{2}-4}}{2}, & r_{4}=\frac{\Delta_{+}-\sqrt{\Delta_{+}{ }^{2}-4}}{2}, \\
r_{2}=\frac{\Delta_{-}+\sqrt{\Delta_{-}{ }^{2}-4}}{2}, & r_{3}=\frac{\Delta_{-}-\sqrt{\Delta_{-}{ }^{2}-4}}{2}
\end{array}
$$

(notice that, by analytic continuation (4) holds for all $\lambda$, i.e. without permuting the indices of the $r_{j}$ 's).

Again it is more natural to view $\Delta_{+}$and $\Delta_{-}$as the branches of the analytic function $\Delta(\lambda)$ "living" (defined) on the two-sheeted Riemann surface of $\sqrt{E(\lambda)}$. We can, then, write

$$
\Delta(\lambda)=\frac{A(\lambda)+\sqrt{E(\lambda)}}{2}
$$


and

$$
r(\lambda)=\frac{\Delta(\lambda)+\sqrt{\Delta(\lambda)^{2}-4}}{2},
$$

where now $\sqrt{\cdot}$ denotes the double-valued square root function. Finally, notice that the above equation implies immediately that

$$
\frac{r^{\prime}(\lambda)}{r(\lambda)}=\frac{\Delta^{\prime}(\lambda)}{\sqrt{\Delta(\lambda)^{2}-4}}
$$

2.2. Floquet Solutions. ¿From the results presented in $[38]$ it follows that $T=T(\xi ; \lambda)$ is similar to a diagonal matrix if and only if $\lambda$ is not a branch point of $r(\lambda)$.

The eigenvectors of $T$ correspond to the (proper) Floquet solutions, namely to the solutions $f_{j}(x ; \lambda), j=1,2,3,4$, of (1) such that

$$
f_{j}(x+b ; \lambda)=r_{j} f_{j}(x ; \lambda) .
$$

Thus, there are four linearly independent Floquet solutions if and only if $T$ is similar to a diagonal matrix. These solutions are defined modulo a constant factor (i.e. a factor which is independent of $x$ ). To fix this ambiguity, for a given real number $\xi$, one introduces (see [27], [11], [14], [36]) the normalized Floquet solutions $\phi_{j}(x)=\phi_{j}(x ; \xi ; \lambda), j=1,2,3,4$, for which

$$
\phi_{j}(\xi ; \xi ; \lambda)=1 .
$$

In case where $\xi=0$, we write

$$
\phi_{j}(x ; \lambda) \stackrel{\text { def }}{=} \phi_{j}(x ; 0 ; \lambda)
$$

and, hence, $\phi_{j}(0 ; \lambda)=1$. In fact we have

$$
\phi_{j}(x ; \xi ; \lambda)=\frac{f_{j}(x ; \lambda)}{f_{j}(\xi ; \lambda)}=\frac{\phi_{j}(x ; 0 ; \lambda)}{\phi_{j}(\xi ; 0 ; \lambda)}=\frac{\phi_{j}(x ; \lambda)}{\phi_{j}(\xi ; \lambda)}
$$

(thus $\phi_{j}(x ; \xi ; \lambda)$ is proportional to $\phi_{j}(x ; \lambda)$ by a factor which is independent of $x$ ).

Again it is more appropriate to view the $\phi_{j}$ 's as the branches of a multivalued $\lambda$-analytic function $\phi(x ; \xi ; \lambda)$. In fact $\phi(x ; \xi ; \lambda)$ is a meromorphic function on $\Gamma$ (the Riemann surface of $r(\lambda))$, whose set of poles is denoted by $\left\{\mu_{n}(\xi)\right\}$. For each $\mu_{n}(\xi)$ there is a $j \in\{1,2,3,4\}$ such that

$$
f_{j}\left(\xi ; \mu_{n}(\xi)\right)=0
$$

(and, as one can see from (15), the normalization (14) is not possible when $\lambda=$ $\left.\mu_{n}(\xi)\right)$.

2.3. Spectrum and Pseudospectrum. ¿From (6)-(7) it follows that the periodic eigenvalues of (1) are the zeros of the entire function

$$
F^{+}(\lambda) \stackrel{\text { def }}{=} F(\lambda ; 0) \stackrel{\text { def }}{=} B(\lambda)-2 A(\lambda)+4
$$

while the antiperiodic eigenvalues of (1) are the zeros of the entire function

$$
F^{-}(\lambda) \stackrel{\text { def }}{=} F(\lambda ; \pi / b) \stackrel{\text { def }}{=} B(\lambda)+2 A(\lambda)+4 \text {. }
$$

Let $\left\{\widetilde{\lambda}_{n}\right\}_{n=0}^{\infty}$ be the set of periodic and antiperiodic eigenvalues of (1), counting (geometric) multiplicities. Then

$$
0=\widetilde{\lambda}_{0}<\tilde{\lambda}_{1} \leq \tilde{\lambda}_{2}<\tilde{\lambda}_{3} \leq \tilde{\lambda}_{4}<\cdots
$$


$\left(\widetilde{\lambda}_{0}, \widetilde{\lambda}_{3}, \widetilde{\lambda}_{4}, \widetilde{\lambda}_{7}, \widetilde{\lambda}_{8}, \ldots\right.$ are the periodic eigenvalues and $\widetilde{\lambda}_{1}, \widetilde{\lambda}_{2}, \widetilde{\lambda}_{5}, \widetilde{\lambda}_{6}, \widetilde{\lambda}_{9}, \ldots$ are the antiperiodic eigenvalues). We thus have (see $[\mathbf{3 8}]$ )

$$
F^{+}(\lambda) F^{-}(\lambda)=-c_{F}^{2} \lambda \prod_{n=1}^{\infty}\left(1-\frac{\lambda}{\widetilde{\lambda}_{n}}\right)
$$

where $c_{F}>0$. The zeros of $F^{+}(\lambda) F^{-}(\lambda)$ can be simple, or double (geometric and algebraic multiplicities agree). The simple positive zeros are the endpoints of the open gaps of the $L_{\rho}^{2}(\mathbb{R})$-spectrum $\sigma_{1}(a, \rho)$ of $(1)$. They are, also, the type I branch points of $r(\lambda)$, i.e. the $\lambda$ 's where $r_{2}(\lambda)=r_{3}(\lambda)= \pm 1,\left|r_{1}(\lambda)\right| \neq 1$. We furthermore have (see (8) and (9))

$$
F^{+}(\lambda) F^{-}(\lambda)=\left[\Delta_{+}(\lambda)^{2}-4\right]\left[\Delta_{-}(\lambda)^{2}-4\right]=\left[r_{1}(\lambda)-r_{4}(\lambda)\right]^{2}\left[r_{2}(\lambda)-r_{3}(\lambda)\right]^{2} .
$$

In $[\mathbf{4 0}]$ we proved that $E(\lambda)$ of (9) has the form

$$
E(\lambda)=c_{E} \lambda \prod_{n=1}^{\infty}\left(1-\frac{\lambda}{\widetilde{\nu}_{n}}\right)
$$

where $c_{E}>0$ and $\widetilde{\nu}_{n}<0$. The zeros of $E(\lambda)$ can be simple, or double. The simple negative zeros are the endpoints of the open $\psi$-gaps of the pseudospectrum (or $\psi$-spectrum) $\sigma_{2}(a, \rho)$ of (1). They are, also, the type II branch points of $r(\lambda)$, i.e. the $\lambda$ 's where $r_{1}(\lambda)=r_{2}(\lambda), r_{3}(\lambda)=r_{4}(\lambda),\left|r_{1}(\lambda)\right| \neq 1$.

Finally, let us mention a (minor) conjecture: The projections on $\mathbb{C}$ of the zeros of $\Delta^{\prime}(\lambda)$ (also of $r^{\prime}(\lambda)$ - see (12)) are real and lie in gaps or $\psi$-gaps.

Assumption I. For the rest of this work we will assume that

$$
F^{+}(\lambda) F^{-}(\lambda)=-c_{F}^{2} \lambda \prod_{n=1}^{2 N}\left(1-\frac{\lambda}{\lambda_{n}}\right) \prod_{n=1}^{\infty}\left(1-\frac{\lambda}{\lambda_{n}^{*}}\right)^{2}
$$

where

$$
0<\lambda_{1}<\lambda_{2}<\cdots<\lambda_{2 N-1}<\lambda_{2 N} .
$$

In other words, we have assumed that the spectrum $\sigma_{1}(a, \rho)$ of (1) has (exactly) $N$ nondegenerate gaps

$$
I_{j}=\left(\lambda_{2 j-1}, \lambda_{2 j}\right), \quad j=1,2, \ldots, N
$$

(notice that the points $\left\{\lambda_{n}^{*}\right\}$ are the "closed" or degenerate gaps of the spectrum). Sometimes the term "gap" will refer to the closed interval $\left[\lambda_{2 j-1}, \lambda_{2 j}\right]$.

For notational convenience we set

$$
P_{F}(\lambda) \stackrel{\text { def }}{=} \lambda \prod_{n=1}^{2 N}\left(\lambda-\lambda_{n}\right) .
$$

Assumption II. For the rest of this work we will assume that

$$
E(\lambda)=c_{E} \lambda \prod_{n=1}^{2 M}\left(1-\frac{\lambda}{\nu_{n}}\right) \prod_{n=1}^{\infty}\left(1-\frac{\lambda}{\nu_{n}^{*}}\right)^{2},
$$

where

$$
0>\nu_{1}>\nu_{2}>\cdots>\nu_{2 M-1}>\nu_{2 M} .
$$


In other words, we have assumed that the $\psi$-spectrum $\sigma_{2}(a, \rho)$ of $(1)$ has (exactly) $M$ nondegenerate $\psi$-gaps

$$
J_{j}=\left(\nu_{2 j}, \nu_{2 j-1}\right), \quad j=1,2, \ldots, M
$$

(notice that the points $\left\{\nu_{n}^{*}\right\}$ are the "closed" or degenerate $\psi$-gaps of the pseudospectrum). Sometimes the term " $\psi$-gap" or "pseudogap" will refer to the closed interval $\left[\nu_{2 j}, \nu_{2 j-1}\right]$.

For notational convenience we set

$$
P_{E}(\lambda) \stackrel{\text { def }}{=} \lambda \prod_{n=1}^{2 M}\left(\lambda-\nu_{n}\right)
$$

and

$$
E(\lambda)=E_{1}(\lambda)^{2} P_{E}(\lambda),
$$

where

$$
E_{1}(\lambda)=c_{1} \prod_{n=1}^{\infty}\left(1-\frac{\lambda}{\nu_{n}^{*}}\right) .
$$

In $[39]$ it was proved that the beam operator $L$ is a perfect square of a second order differential operator (i.e. $a(x) \rho(x) \equiv$ const.) if and only if $M=0$, namely the pseudospectrum has no $\psi$-gaps (equivalently $E(\lambda) \lambda^{-1}$ is the square of an entire function-see (23)).

Remark 2. The question of existence of beam operators satisfying Assumptions I and II, i.e. with exactly $N$ nondegenerate gaps in its spectrum and $M>0$ nondegenerate $\psi$-gaps in its pseudospectrum remains open (if $M=0$, then $L$ is the square of a Hill operator and it is well-known that such operators exist). However we can observe the following:

Under Assumption I (16) and (17) give

$$
B(\lambda)+2 A(\lambda)+4=\lambda P_{1}(\lambda) U(\lambda)^{2}
$$

and

$$
B(\lambda)-2 A(\lambda)+4=P_{2}(\lambda) V(\lambda)^{2},
$$

where $U(\lambda)$ and $V(\lambda)$ are entire functions of order $1 / 4$ whose zeros are positive, simple, satisfying certain interlacing restrictions, while (see (22))

$$
\lambda P_{1}(\lambda) P_{2}(\lambda)=P_{F}(\lambda) .
$$

Likewise, under Assumption II (9) gives

$$
E(\lambda)=A(\lambda)^{2}-4 B(\lambda)=P_{E}(\lambda) W(\lambda)^{2},
$$

where $W(\lambda)$ is entire of order $1 / 4$ whose zeros are negative and simple, while the polynomial $P_{E}(\lambda)$ is given by (25). By eliminating $A$ and $B$ from (28), (29), and (30) we get the following "Diophantine-like" equation for $U, V$, and $W$ :

$$
\frac{\left(\lambda P_{1} U^{2}-P_{2} V^{2}\right)^{2}}{16}-2 \lambda P_{1} U^{2}-2 P_{2} V^{2}+16=P_{E} W^{2} .
$$

If, given polynomials $P_{1}(\lambda), P_{2}(\lambda)$, and $P_{E}(\lambda)$, equation (31) has a solution $U(\lambda), V(\lambda), W(\lambda)$, then one can write down an $A(\lambda)$ and a $B(\lambda)$, but still this does not answer the question of existence of a beam operator satisfying Assumptions I and II. 
2.4. The Tilded Equation. Let us assume here that $a(x), \rho(x) \in C^{2}(\mathbb{R})$. In connection with (1) let us consider the Euler-Bernoulli equation

$$
\left[\widetilde{a}(x) u^{\prime \prime}(x)\right]^{\prime \prime}=\lambda \widetilde{\rho}(x) u(x),
$$

where

$$
\widetilde{a}(x)=\frac{1}{\rho(x)}, \quad \text { and } \quad \widetilde{\rho}(x)=\frac{1}{a(x)}
$$

in particular

$$
\frac{\widetilde{\rho}(x)}{\widetilde{a}(x)}=\frac{\rho(x)}{a(x)},
$$

thus $\widetilde{a}(x)$ and $\widetilde{\rho}(x)$ satisfy the normalization (2). If $Q$ is a quantity related to (1), we denote by $\widetilde{Q}$ the corresponding quantity of $(32)$. Observe that $\widetilde{u}(x)$ is a solution of (32) if and only if $\widetilde{u}(x)=a(x) u^{\prime \prime}(x)$, where $u(x)$ is a solution of (1). In particular, if $f_{j}(x)$ is a Floquet solution of (1) with Floquet multiplier $r_{j}$, then $a(x) f_{j}^{\prime \prime}(x)$ is a Floquet solution of (32) with the same Floquet multiplier $r_{j}$. Hence, (1) and (32) have the same Floquet multipliers, which gives (see (5))

$$
\widetilde{A}(\lambda)=A(\lambda), \quad \widetilde{B}(\lambda)=B(\lambda),
$$

and hence (see (9))

$$
\widetilde{E}(\lambda)=E(\lambda) .
$$

It other words, (1) and (32) have the same spectrum and pseudospectrum.

2.5. A Multipoint Eigenvalue Problem. In [38] we introduced the following multipoint problem as the analog of Hill's Dirichlet problem for the EulerBernoulli case:

(34) $\left[a(x) u^{\prime \prime}(x)\right]^{\prime \prime}=\lambda \rho(x) u(x), \quad u(\xi)=u(\xi+b)=u(\xi+2 b)=u(\xi+3 b)=0$,

where $\xi \in \mathbb{R}$ is a given point. An eigenvalue of (34) is any value of $\lambda$ for which (34) has a nontrivial solution. We call such a solution an eigenfunction of (34). Physically the above problem describes the vibration of a beam fixed at the four points $\xi, \xi+b, \xi+2 b, \xi+3 b$.

Let $u_{j}(x ; \xi ; \lambda), j=1,2,3,4$, be the fundamental solutions of (1) with respect to $\xi$. Since every solution of (1) is a linear combination of the fundamental solutions, it follows that $\lambda$ is an eigenvalue of (34) if and only if $\lambda$ is a zero of the function

$$
H(\xi ; \lambda)=\left|\begin{array}{ccc}
u_{2}(\xi+b ; \xi ; \lambda) & u_{3}(\xi+b ; \xi ; \lambda) & u_{4}(\xi+b ; \xi ; \lambda) \\
u_{2}(\xi+2 b ; \xi ; \lambda) & u_{3}(\xi+2 b ; \xi ; \lambda) & u_{4}(\xi+2 b ; \xi ; \lambda) \\
u_{2}(\xi+3 b ; \xi ; \lambda) & u_{3}(\xi+3 b ; \xi ; \lambda) & u_{4}(\xi+3 b ; \xi ; \lambda)
\end{array}\right|
$$

(of course $H(\xi ; \lambda)$ is entire in $\lambda$ of order $1 / 4$ ). We can, thus, say that the spectrum of $(34)$ is the set of zeros of $H(\xi ; \lambda)$. A simple calculation gives

$$
H(\xi ; 0)=b^{4}\left(\int_{0}^{b} \frac{d x}{a(x)}\right)^{2}>0,
$$

thus 0 is not an eigenvalue of (34) and

$$
H(\xi ; \lambda)=b^{4}\left(\int_{0}^{b} \frac{d x}{a(x)}\right)^{2} \prod_{m}\left[1-\frac{\lambda}{\zeta_{m}(\xi)}\right],
$$

where $\left\{\zeta_{m}(\xi)\right\}_{m}$ is the "spectrum" of (34). 
The theorem below should be compared with the property of the Hill operator stating that the Dirichlet eigenvalues are simple and their corresponding eigenfunctions are Floquet solutions.

Theorem A. Let $\zeta$ be an eigenvalue of (34) and $V(\zeta)$ the corresponding eigenspace, namely the vector space of all eigenfunctions of (34) associated to $\zeta$. Then $\operatorname{dim} V(\zeta)=1$ or 2 . If $\operatorname{dim} V(\zeta)=1$, then $V(\zeta)$ is spanned by a (proper) Floquet solution; if $\operatorname{dim} V(\zeta)=2$, then $V(\zeta)$ is spanned by two (proper) Floquet solutions, one belonging to $L^{2}(-\infty, 0)$ and the other belonging to $L^{2}(0, \infty)$.

The main result regarding the spectrum of $(35)$, i.e. the zeros of $H(\xi ; \lambda)$, is the following:

Theorem B. All zeros of $H(\xi ; \lambda)$, of $(35)$, are real and they are located as follows: (a) $H(\xi ; \lambda)$ has exactly one (simple) zero in (the closure of) each gap of the spectrum $\sigma_{1}(a, \rho)$ (with the understanding that, if the gap is degenerate, i.e. collapses to a double periodic or antiperiodic eigenvalue, say $\lambda^{*}$, then the simple zero of $H(\xi ; \lambda)$ is $\lambda^{*}$, and it is, of course, independent of $\left.\xi\right)$; (b) $H(\xi ; \lambda)$ has exactly two zeros (counting multiplicities) in (the closure of) each $\psi$-gap of the pseudospectrum. In case (b), if the $\psi$-gap is degenerate, i.e. collapses to a point $\nu^{*}$, then $\nu^{*}$ is a double zero of $H(\xi ; \lambda)$, for any $\xi$. There are no other zeros of $H(\xi ; \lambda)$.

The proofs of the above theorems can be found in [38].

2.6. The Poles of $\phi(x ; \xi ; \lambda)$. The value $\lambda=\lambda_{0}=0$ is very special. The Floquet multiplier $r(\lambda)$ has a fourth root branch point there and there is only one proper (normalized) Floquet solution, namely $\phi_{j}(x ; \xi ; 0)=1, j=1,2,3,4$.

As a function of $\lambda, \phi(x ; \xi ; \lambda)$ is meromorphic on $\Gamma$ (see Subsection 2.2). Theorems A and B imply that each pole $\mu_{n}(\xi)$ of $\phi(x ; \xi ; \lambda)$ is simple and its projection on $\mathbb{C}$ (since $\mu_{n}(\xi)$ lives on $\Gamma$ ) must be a zero of $H(\xi ; \lambda)$. In fact, each zero of $H(\xi ; \lambda)$ in a nondegenerate gap or $\psi$-gap is the projection of a $\mu_{n}(\xi)$. Even if $H(\xi ; \lambda)$ has a double zero $\zeta$ in a nondegenerate $\psi$-gap, $\phi(x ; \xi ; \lambda)$ has two simple poles (lying on different sheets of $\Gamma$ ) whose projection on $\mathbb{C}$ is $\zeta$. However, a zero $\zeta$ of $H(\xi ; \lambda)$ in a degenerate gap or $\psi$-gap is not the projection of a pole of $\phi(x ; \xi ; \lambda)$ : If $\zeta$ is such a zero, then $\zeta$ is not a branch point of $r(\lambda)$ and there are four linearly independent Floquet solutions $f_{j}(x ; \zeta), j=1,2,3,4$, which can be chosen so that $f_{j}(\xi ; \zeta) \neq 0$, for all $j$.

Thus each nondegenerate gap contains (the projection of) exactly one $\mu_{n}(\xi)$, while each nondegenerate $\psi$-gap contains (the projection of) exactly two $\mu_{n}(\xi)$ 's. There is no other possibility for the poles of $\phi(x ; \xi ; \lambda)$. If we introduce the polynomial (in $\lambda$ )

$$
P(\xi ; \lambda)=\prod_{n=-2 M, n \neq 0}^{N}\left[1-\frac{\lambda}{\mu_{n}(\xi)}\right],
$$

(where $\mu_{-2 M}(\xi), \mu_{-(2 M-1)}(\xi)$ are in $\left[\nu_{2 M}, \nu_{2 M-1}\right]$ and so on, and finally $\mu_{N}(\xi)$ is in $\left[\lambda_{2 N-1}, \lambda_{2 N}\right]$, i.e. two $\mu_{n}(\xi)$ 's in the closure of each nondegenerate $\psi$-gap and one $\mu_{n}(\xi)$ in the closure of each nondegenerate spectral gap) then, the above discussion yields that

$$
H(\xi ; \lambda)=c(\lambda) P(\xi ; \lambda),
$$


where $c(\lambda)$ is the entire function (see (36), (20), and (23))

$$
c(\lambda)=b^{4}\left(\int_{0}^{b} \frac{d x}{a(x)}\right)^{2} \prod_{n=1}^{\infty}\left[1-\frac{\lambda}{\lambda_{n}^{*}}\right] \prod_{n=1}^{\infty}\left[1-\frac{\lambda}{\nu_{n}^{*}}\right]^{2} .
$$

The sets $\left\{\lambda_{n}^{*}\right\}$ and $\left\{\nu_{n}^{*}\right\}$ are, of course, independent of $\xi$, hence so is $c(\lambda)$.

Remark 3. Since (see (15))

$$
\phi(x ; \xi ; \lambda)=\frac{\phi(x ; \lambda)}{\phi(\xi ; \lambda)},
$$

it follows that the $\mu_{n}(\xi)$ 's, $\xi \neq 0$, are the zeros of $\phi(\xi ; \lambda)$ (of course, the $\mu_{n}(0)$ 's are, by definition, the poles of $\phi(\xi ; \lambda))$.

In [14] it is suggested that the (periodic) inverse spectral data for $L$ is the Riemann surface $\Gamma$ (which is determined by the branch points of $r(\lambda)$ ) together with the set of poles $\left\{\mu_{n}(0)\right\}_{n=-2 M, n \neq 0}^{N}$ of $\phi(x ; \lambda)$ (notice that each $\mu_{n}(0)$ is a point on $\Gamma$, i.e. $\mu_{n}(0)$ is not just a complex number, since it also contains the information: on which sheet of $\Gamma$ does it lie). This is, of course, inspired by the inverse theory of the Hill's operator (see, e.g., [13], [14], [15], [30], [31], [48]).

For the Euler-Bernoulli operator, we need both the $L_{\rho}^{2}(\mathbb{R})$-spectrum and the pseudospectrum in order to determine the Riemann surface $\Gamma$ and, hence, the intervals in which the $\mu_{n}$ 's are confined. This is why the pseudospectrum plays an essential role in the Euler-Bernoulli inverse spectral theory. However, we notice that, if we know the periodic and antiperiodic eigenvalues $\left\{\widetilde{\lambda}_{n}\right\}_{n \geq 1}$, then (see (18)) we can determine the entire functions $F(\lambda ; 0)$ and $F(\lambda ; \pi / b)$. It follows (see (16) and (17)) that $A(\lambda)$ and $B(\lambda)$, and therefore $E(\lambda)$ can be determined. Thus, if the spectrum has no degenerate gaps, then it determines the pseudospectrum. However, if there are degenerate gaps, the spectrum (as a subset of $\mathbb{R}$ ) may not be enough to determine the pseudospectrum. If, for example, the spectrum is $[0, \infty)$, we believe that $a(x)$ and $\rho(x)$ are not necessarily constant and, hence, the pseudospectrum is not uniquely determined.

2.7. Asymptotics as $|\lambda| \rightarrow \infty$. We divide the complex $\lambda$-plane into 8 closed sectors $S_{l}, l=0,1, \ldots, 7$, defined by

$$
\frac{l \pi}{4} \leq \arg (\lambda) \leq \frac{(l+1) \pi}{4} .
$$

Then, for each $S_{l}$ (see [35], Part I, Chap. II), there are four linearly independent solutions $v_{j l}(x ; \lambda), j=1,2,3,4$, of $(1)$, analytic for $\lambda \in S_{l}$, such that, given $x_{0}>0$,

$$
\left|v_{j l}(x ; \lambda)-\frac{e^{\varepsilon_{j} \lambda^{1 / 4} S(x, 0)}}{\rho(x)^{3 / 8} a(x)^{1 / 8}}\right| \leq K \frac{\left|e^{\varepsilon_{j} \lambda^{1 / 4} S(x, 0)}\right|}{|\lambda|^{1 / 4}}, \quad 0 \leq x \leq x_{0},
$$

where

$$
S(x ; \xi)=\int_{\xi}^{x}\left[\frac{\rho(y)}{a(y)}\right]^{1 / 4} d y
$$

(in particular, due to normalization $(2), S(n b, 0)=n b$, if $n \in \mathbb{Z}$ ). Here, $\lambda^{1 / 4}$ stands for the principal branch of the fourth root (so that $\Re\left\{\lambda^{1 / 4}\right\} \geq 0, \Im\left\{\lambda^{1 / 4}\right\} \geq 0$ ), $\left\{\varepsilon_{1}, \varepsilon_{2}, \varepsilon_{3}, \varepsilon_{4}\right\}=\{i,-1,-i, 1\}$, and the (positive) constant $K$ depends on $a(x)$, $\rho(x)$, and $x_{0}$. Similar formulas hold for the $x$-derivatives of $v_{j l}(x ; \lambda)$. 
We now present some consequences of the above formulas. If $r_{j}(\lambda), j=$ $1,2,3,4$, are the Floquet multipliers of (1), then

$$
\left|\frac{r_{j}(\lambda)}{e^{\varepsilon_{j} \lambda^{1 / 4} b}}-1\right| \leq \frac{K}{|\lambda|^{1 / 4}}, \quad j=1,2,3,4,
$$

where $\lambda^{1 / 4}$ is the principal branch of the fourth root, $\varepsilon_{1}=1, \varepsilon_{2}=i, \varepsilon_{3}-i, \varepsilon_{4}=-1$, and the constant $K>0$ depends on $a(x), \rho(x)$, but not on $\lambda$.

If $\phi_{j}(x ; \lambda), j=1,2,3,4$, are the normalized Floquet solutions introduced in Section 2.1, then (see [37]) we have, as $|\lambda| \rightarrow \infty$, uniformly in $x \in\left[0, x_{0}\right]$,

$$
\phi_{j}(x ; \lambda)=\frac{\rho(0)^{3 / 8} a(0)^{1 / 8}}{\rho(x)^{3 / 8} a(x)^{1 / 8}} e^{\varepsilon_{j} \lambda^{1 / 4} S(x, 0)+\varepsilon_{j}^{-1} \lambda^{-1 / 4} \widetilde{S}(x)}\left[1+O\left(\frac{1}{\sqrt{\lambda}}\right)\right],
$$

where

$$
\widetilde{S}(x)=\int_{0}^{x} \frac{10 a a^{\prime} \rho \rho^{\prime}-13\left(a^{\prime}\right)^{2} \rho^{2}-45 a^{2}\left(\rho^{\prime}\right)^{2}+24 a a^{\prime \prime} \rho^{2}+40 a^{2} \rho \rho^{\prime \prime}}{128 a^{7 / 4} \rho^{9 / 4}},
$$

$\varepsilon_{1}=1, \varepsilon_{2}=i, \varepsilon_{3}-i$, and $\varepsilon_{4}=-1$. However here

$$
\delta \leq \arg \lambda \leq \pi-\delta \quad \text { or } \quad \pi+\delta \leq \arg \lambda \leq 2 \pi-\delta,
$$

where $\delta>0$ is any given constant. The quantities $S(x, 0), K$, and $x_{0}$ are as before, but $K$ and $x_{0}$ depend on $\delta$. Under the assumption of finitely many gaps and finitely many $\psi$-gaps, i.e. under (20) and (23), (43) is valid for all directions without the restriction of (45). Similar formulas exist for the derivatives of $\phi_{j}(x ; \lambda)$. Furthermore, we have, as $|\lambda| \rightarrow \infty$,

$$
\frac{\phi_{j}^{\prime}(x ; \lambda)}{\phi_{j}(x ; \lambda)}=\varepsilon_{j} \frac{\rho(x)^{1 / 4}}{a(x)^{1 / 4}} \lambda^{1 / 4}+\text { l.o.t. }
$$

and (notice that derivatives with respect to $\lambda$ can be written as integrals via Cauchy's formula)

$$
\frac{1}{\phi_{j}(x ; \lambda)} \frac{d}{d \lambda}\left[\phi_{j}(x ; \lambda)\right]=\frac{\varepsilon_{j}}{4} \lambda^{-3 / 4} S(x, 0)-\frac{\varepsilon_{j}^{-1}}{4} \lambda^{-5 / 4} \widetilde{S}(x)+\text { l.o.t. },
$$

where "l.o.t." stands for "lower order terms" and means terms whose magnitude is less than the magnitude of the leading term by a factor of (at least) $\lambda^{-1 / 4}$. Setting $x=b$ in the last equation and invoking (41), and (2) one obtains

$$
\frac{r_{j}^{\prime}(\lambda)}{r_{j}(\lambda)}=\frac{\varepsilon_{j} b}{4} \lambda^{-3 / 4}+\text { l.o.t. }
$$

which in turn gives (see (2))

$$
\left|\frac{\Delta^{\prime}(\lambda)}{\sqrt{\Delta(\lambda)^{2}-4}}\right|=\frac{b}{4}|\lambda|^{-3 / 4}+\text { l.o.t.. }
$$

Remark 4. The asymptotic formulas presented in this subsection hold for general $a(x)$ and $\rho(x)$, i.e. even when the Assumptions I and II do not hold. However, if Assumptions I and II do hold, then the formulas (46), (47), and (48) imply that the functions

$$
\frac{\phi^{\prime}(x ; \lambda)}{\phi(x ; \lambda)}, \quad \frac{1}{\phi(x ; \lambda)} \frac{d}{d \lambda}[\phi(x ; \lambda)], \quad \text { and } \quad \frac{r^{\prime}(\lambda)}{r(\lambda)}
$$


are meromorphic on $\bar{\Gamma}=\Gamma \cup\{$ points at $\infty\}$, hence $\bar{\Gamma}$ is a compact Riemann surface (and, therefore, the Riemann surface of an algebraic function - see, e.g., [45]). Since $r(\lambda) \neq 0$ on $\Gamma$, it follows that $r^{\prime}(\lambda) / r(\lambda)$ can have poles only at the branch points of $r(\lambda)$. In fact, for $\lambda \neq 0, r(\lambda)$ has only square-root branch points, and hence $r^{\prime}(\lambda) / r(\lambda)$ can only have simple poles at these points. In Subsection 3.1 we will derive a (purely) algebraic equation for $r^{\prime}(\lambda) / r(\lambda)$.

If $u_{1}(x ; \xi ; \lambda)$ is the first fundamental solution of (1) with respect to $\xi$, then

$$
\begin{gathered}
u_{1}(x ; \xi ; \lambda)=\frac{a(\xi)^{1 / 8} \rho(\xi)^{3 / 8}}{2 a(x)^{1 / 8} \rho(x)^{3 / 8}} \cdot\left\{\cosh \left[\lambda^{1 / 4} S(x ; \xi)\right]+\cos \left[\lambda^{1 / 4} S(x ; \xi)\right]\right\}+\text { l.o.t., } \\
u_{1}^{\prime}(x ; \xi ; \lambda)=\frac{\lambda^{1 / 4} a(\xi)^{1 / 8} \rho(\xi)^{3 / 8}}{2 a(x)^{3 / 8} \rho(x)^{1 / 8}} \cdot\left\{\sinh \left[\lambda^{1 / 4} S(x ; \xi)\right]-\sin \left[\lambda^{1 / 4} S(x ; \xi)\right]\right\}+\text { l.o.t., } \\
u_{1}^{\prime \prime}(x ; \xi ; \lambda)=\frac{\lambda^{1 / 2} a(\xi)^{1 / 8} \rho(\xi)^{3 / 8}}{2 a(x)^{5 / 8} \rho(x)^{-1 / 8}} \cdot\left\{\cosh \left[\lambda^{1 / 4} S(x ; \xi)\right]-\cos \left[\lambda^{1 / 4} S(x ; \xi)\right]\right\}+\text { l.o.t., } \\
a(x) u_{1}^{\prime \prime \prime}(x ; \xi ; \lambda)=\frac{\lambda^{3 / 4} a(\xi)^{1 / 8} \rho(\xi)^{3 / 8}}{2 a(x)^{-1 / 8} \rho(x)^{-3 / 8}} \cdot\left\{\sinh \left[\lambda^{1 / 4} S(x ; \xi)\right]+\sin \left[\lambda^{1 / 4} S(x ; \xi)\right]\right\}+\text { l.o.t., }
\end{gathered}
$$

as $|\lambda| \rightarrow \infty$, where primes denote derivatives with respect to $x$, as usual; $x$ and $\xi$ are in a bounded interval and $S(x ; \xi)$ is given by (41). Furthermore $\lambda$ satisfies

$$
\pi+\delta \leq \arg \lambda \leq 2 \pi-\delta,
$$

which is a little weaker condition than (45). Similar relations hold for the other fundamental solutions.

¿From the above it follows that, if $E(\lambda), F^{+}(\lambda), F^{-}(\lambda)$, and $H(\xi ; \lambda)$ are the functions defined by (9), (16), (17) and (35) respectively, then, under (45),

and

$$
\begin{gathered}
E(\lambda)=4\left[\cosh \left(\lambda^{1 / 4} b\right)-\cos \left(\lambda^{1 / 4} b\right)\right]^{2}+\text { l.o.t. } \\
F^{+}(\lambda) F^{-}(\lambda)=-16 \sinh ^{2}\left(\lambda^{1 / 4} b\right) \sin ^{2}\left(\lambda^{1 / 4} b\right)+\text { l.o.t. }
\end{gathered}
$$

$$
H(\xi ; \lambda)=\frac{\sinh \left(\lambda^{1 / 4} b\right) \sin \left(\lambda^{1 / 4} b\right)}{\lambda^{3 / 2} a(\xi)^{1 / 2} \rho(\xi)^{3 / 2}} \cdot\left\{\cosh \left(\lambda^{1 / 4} b\right)-\cos \left(\lambda^{1 / 4} b\right)\right\}^{2}+\text { l.o.t. }
$$

where, as usual "l.o.t." stands for "lower order terms" as explained earlier.

\section{The Riemann Surface $\bar{\Gamma}$}

Let $\mathbb{C}_{1}, \mathbb{C}_{2}, \mathbb{C}_{3}$, and $\mathbb{C}_{4}$, be four copies of the complex plane. We will show how to "cut and paste" these copies in order to construct the Riemann surface $\Gamma$. We prefer to attach the points at infinity to these complex planes and construct a slightly extended and compactified surface $\bar{\Gamma}$ (see Remark 4 ). We, thus start by consider the (Riemann) spheres (also known as projective lines)

$$
\Sigma_{j}=\mathbb{C}_{j} \cup\left\{\infty_{j}\right\}, \quad j=1,2,3,4 .
$$

Next, (a) on each $\Sigma_{j}$ we consider the cuts along the intervals

$$
\bar{J}_{j}=\left[\nu_{2 j}, \nu_{2 j-1}\right], \quad j=1,2, \ldots, M ;
$$

(b) on $\Sigma_{2}$ and $\Sigma_{3}$ we consider the cuts along the intervals

$$
\bar{I}_{j}=\left[\lambda_{2 j-1}, \lambda_{2 j}\right], \quad j=1,2, \ldots, N ;
$$


finally (c) on each $\Sigma_{j}$ we consider a cut $C$ joining $0_{j}$ to $\infty_{j}$, so that $C$ does not intersect any of the $\bar{J}_{j}$ 's nor $\bar{I}_{j}$ 's. We join $\Sigma_{1}$ and $\Sigma_{2}$ along each $\bar{J}_{j}$ (so that each $\nu_{j}$ behaves as a square-root branch point for $\Sigma_{1}$ and $\Sigma_{2}$ ), we join $\Sigma_{3}$ and $\Sigma_{4}$ along each $\bar{J}_{j}$ (so that each $\nu_{j}$ behaves as a square-root branch point for $\Sigma_{3}$ and $\Sigma_{4}$ ), we join $\Sigma_{2}$ and $\Sigma_{3}$ along each $\bar{I}_{j}$ (so that each $\lambda_{j}$ behaves as a square-root branch point for $\Sigma_{2}$ and $\left.\Sigma_{3}\right)$, and, finally, we join $\Sigma_{1}$ to $\Sigma_{2}, \Sigma_{2}$ to $\Sigma_{4}$, and $\Sigma_{4}$ to $\Sigma_{1}$ along $C$ (so that 0 and $\infty$ behave as fourth-root branch points for $\Sigma_{1}, \Sigma_{2}, \Sigma_{3}$, and $\Sigma_{4}$ ). This is how $\bar{\Gamma}$ is constructed (we notice that $\bar{\Gamma}$ agrees with the general description of such surfaces - see [11]). By applying the Riemann-Hurwitz formula (see, e.g., [29], or [45], Sec. 10-6) it is easy to see that the genus $g$ of $\bar{\Gamma}$ is

$$
g=2 M+N .
$$

Remark 5. As we have seen, the normalized Floquet solution $\phi(x ; \xi ; \lambda)$ of (1) is meromorphic (with respect to $\lambda$ ) on $\Gamma$ (and not on $\bar{\Gamma}$ ). However, e.g., $\phi^{\prime}(x ; \lambda) / \phi(x ; \lambda)$ (see Remark 4) is meromorphic on $\bar{\Gamma}$. The number of poles (and, also, the number of zeros) of $\phi(x ; \xi ; \lambda)$ is equal to the genus $g$ (see Subsection 2.5). As we will see, this fact plays a very important role in the solution of the inverse problem. We expect that this property is generically valid for periodic ordinary differential operators of any order (including matrix-valued operators).

Let (i) $\alpha_{j}^{\prime}$ be a loop around $\bar{J}_{j}, j=1, \ldots, M$, on $\Sigma_{1}$ and $\Sigma_{2}$, (ii) $\alpha_{j}^{\prime \prime}$ be a loop around $\bar{J}_{j}, j=1, \ldots, M$, on $\Sigma_{3}$ and $\Sigma_{4}$, (iii) $\alpha_{j}$ be a loop around $\bar{I}_{j}, j=1, \ldots, N$, on $\Sigma_{2}$ and $\Sigma_{3}$, (iv) $\beta_{j}^{\prime}$ be a loop around $\left[0, \nu_{2 j-1}\right], j=1, \ldots, M$, on $\Sigma_{1}$ and $\Sigma_{2}$, (v) $\beta_{j}^{\prime \prime}$ be a loop around $\left[0, \nu_{2 j-1}\right], j=1, \ldots, M$, on $\Sigma_{3}$ and $\Sigma_{4}$, and finally (vi) $\beta_{j}$ be a loop around $\left[0, \lambda_{2 j-1}\right], j=1, \ldots, N$, on $\Sigma_{2}$ and $\Sigma_{3}$. Then, these loops form a homology basis for $\bar{\Gamma}$ (see, e.g., $[\mathbf{4 5}]$ ).

3.1. A Characterization of $\bar{\Gamma}$ via $r^{\prime}(\lambda) / r(\lambda)$. In Remark 4 it was pointed out that the quantity

$$
s(\lambda) \stackrel{\text { def }}{=} \frac{r^{\prime}(\lambda)}{r(\lambda)}
$$

is meromorphic on $\bar{\Gamma}$. Here we want to derive a (purely) algebraic equation for $s(\lambda)$. This will give us a better understanding of $\bar{\Gamma}$, since the equation (5) is not algebraic.

The equation that $r(\lambda)$ satisfies, namely (5), remains invariant under the transformation

$$
r(\lambda) \mapsto r(\lambda)^{-1}
$$

and, of course, the same is true for $\Delta(\lambda)=r(\lambda)+r(\lambda)^{-1}$. On the other hand, under the transformation (53) we have that

$$
\ln r(\lambda) \mapsto-\ln r(\lambda)
$$

and hence

$$
s(\lambda) \mapsto-s(\lambda) .
$$

It follows that $s(\lambda)$ must satisfy a "biquadratic" equation, i.e. an equation of the form

$$
s(\lambda)^{4}-p_{1}(\lambda) s(\lambda)^{2}+p_{2}(\lambda)=0,
$$


where $p_{1}(\lambda)$ and $p_{2}(\lambda)$ are rational functions of $\lambda$. The poles of $p_{1}(\lambda)$ and $p_{2}(\lambda)$ can be located only at the poles of $r^{\prime}(\lambda) / r(\lambda)$, namely at the zeros of $P_{E}(\lambda) P_{F}(\lambda)$.

Using (12) in (52) one gets immediately that

$$
s(\lambda)^{2}=\frac{\Delta^{\prime}(\lambda)^{2}}{\Delta(\lambda)^{2}-4},
$$

which, in turn, implies that the Riemann surface of $s(\lambda)^{2}$ is the hyperelliptic surface of $\sqrt{P_{E}(\lambda)}$.

To determine $p_{1}(\lambda)$ and $p_{2}(\lambda)$, we first observe that (10) gives immediately

$$
\left(\Delta^{\prime}\right)^{2}=\frac{4\left(A^{\prime}\right)^{2} E+\left(E^{\prime}\right)^{2}+4 A^{\prime} E^{\prime} \sqrt{E}}{16 E}
$$

and

It follows that (see (19))

$$
\Delta^{2}-4=\frac{(A+\sqrt{E})^{2}-16}{4} .
$$

$$
s(\lambda)^{2}=\frac{\left[4\left(A^{\prime}\right)^{2} E+\left(E^{\prime}\right)^{2}+4 A^{\prime} E^{\prime} \sqrt{E}\right]\left[\left(A^{2}+E-16\right)-2 A \sqrt{E}\right]}{64 E F_{+} F_{-}},
$$

or

$$
\begin{gathered}
s(\lambda)^{2}=\frac{\left[4\left(A^{\prime}\right)^{2} E+\left(E^{\prime}\right)^{2}\right]\left(A^{2}+E-16\right)-8 A A^{\prime} E^{\prime} E}{64 E F_{+} F_{-}} \\
+\frac{2 A^{\prime} E^{\prime}\left(A^{2}+E-16\right)-A\left[4\left(A^{\prime}\right)^{2} E+\left(E^{\prime}\right)^{2}\right]}{32 E F_{+} F_{-}} \sqrt{E}
\end{gathered}
$$

Hence

$$
p_{1}(\lambda)=\frac{\left[4\left(A^{\prime}\right)^{2} E+\left(E^{\prime}\right)^{2}\right]\left(A^{2}+E-16\right)-8 A A^{\prime} E^{\prime} E}{32 E F_{+} F_{-}}
$$

and

$$
\begin{gathered}
p_{2}(\lambda)=\frac{\left\{\left[4\left(A^{\prime}\right)^{2} E+\left(E^{\prime}\right)^{2}\right]\left(A^{2}+E-16\right)-8 A A^{\prime} E^{\prime} E\right\}^{2}}{\left(64 E F_{+} F_{-}\right)^{2}} \\
-\frac{\left\{2 A^{\prime} E^{\prime}\left(A^{2}+E-16\right)-A\left[4\left(A^{\prime}\right)^{2} E+\left(E^{\prime}\right)^{2}\right]\right\}^{2}}{\left(32 F_{+} F_{-}\right)^{2} E}
\end{gathered}
$$

\section{A Brief Review of the Inverse Hill Theory}

Here we review briefly the inverse spectral theory of the Hill equation

$$
H u=-u^{\prime \prime}+q(x) u=\lambda u, \quad-\infty<x<\infty,
$$

where

$$
q(x+b)=q(x) .
$$

For the details, one can see, e.g., [13].

The Floquet multipliers $r_{1}, r_{2}$, are the branches of the double-valued analytic function $r(\lambda)$ satisfying

$$
r^{2}-\Delta_{H}(\lambda) r+1=0
$$


where $\Delta_{H}(\lambda)$ is the well-known discriminant of $H$ (i.e. the trace of the associated Floquet matrix). Let us assume that

$$
\Delta_{H}(\lambda)^{2}-4=C \prod_{n=0}^{2 N}\left(1-\frac{\lambda}{\lambda_{n}}\right) \prod_{n=1}^{\infty}\left(1-\frac{\lambda}{\lambda_{n}^{*}}\right)^{2},
$$

where

$$
\lambda_{0}<\lambda_{1}<\lambda_{2}<\cdots<\lambda_{2 N-1}<\lambda_{2 N}
$$

i.e. the spectrum $\sigma$ of $H$ has (exactly) $N$ nondegenerate gaps. It is then natural to introduce the polynomial

$$
R(\lambda)=\prod_{j=0}^{2 N}\left(\lambda-\lambda_{j}\right),
$$

so that the Riemann surface $S$ of $r(\lambda)$ is the hyperelliptic surface of $\sqrt{R(\lambda)}$ without the point at $\infty$. The genus of the compactified surface $\bar{S}$ is, of course $N$.

Next, let $\chi_{j}(x ; \xi ; \lambda), j=1,2$, be the normalized Floquet solutions (i.e. $\chi_{j}(\xi ; \xi ; \lambda)=$ $1)$. These two solutions are the branches of a double-valued $\lambda$-meromorphic function $\chi(x ; \xi ; \lambda)$, living on $S$. The function $\chi(x ; \xi ; \lambda)$ has $N$ simple poles $\left\{\tau_{n}(\xi)\right\}_{n=1}^{N}$, one in each nondegenerate gap.

The key to the solution of the periodic inverse spectral problem is the system of the evolution equations of the poles $\left\{\tau_{n}(\xi)\right\}_{n=1}^{N}$ (also known as the Dubrovin equations), namely

$$
\frac{d \tau_{n}}{d \xi}=-\frac{2 i \sqrt{R\left(\tau_{n}\right)}}{\prod_{k \neq n}\left(\tau_{n}-\tau_{k}\right)}, \quad n=1,2, \ldots, N
$$

where the sign of the root, at $\xi=0$, is determined from the sheet of $\tau_{n}(0)$. Thus $\left\{\tau_{n}(0)\right\}_{n=1}^{N}$ determines $\left\{\tau_{n}(\xi)\right\}_{n=1}^{N}$, for all $\xi$.

Knowing the $\tau_{n}(\xi)$ 's, one reconstructs the potential $q(x)$ by using the trace formula

$$
q(x)=\lambda_{0}+\sum_{n=1}^{N} \lambda_{2 n-1}+\lambda_{2 n}-2 \tau_{n}(x) .
$$

Alternatively, one can use the fact that

$$
\widehat{\chi}(x ; \lambda)=\frac{1}{\chi(x ; \lambda)} \frac{\partial \chi(x ; \lambda)}{\partial \lambda}
$$

is meromorphic on $\bar{S}$ with known poles and residues (see the above remark). Thus $\widehat{\chi}(x ; \lambda)$ can be recovered, and then $\chi(x ; \lambda)$ (with the help of its asymptotics, as $|\lambda| \rightarrow \infty)$. From $\chi(x ; \lambda)$ one gets $q(x)$ immediately.

There is, yet, a third way to recover $q$. The Baker-Akhiezer function $\chi(x ; \lambda)$ can be written (via theta functions) in terms of its zeros, i.e. the $\tau_{n}(x)$ 's, its poles, i.e. the $\tau_{n}(0)$ 's, and the differentials of $S$ (see [14]). Then we can substitute this expression for $\chi(x ; \lambda)$ in $(55)$ and obtain $q$.

All derivations of the evolution equations (56) which can be found in the existing literature use equation (55). It seems hopeless that any of such derivations can be extended to higher-order equations. Hence, a different approach is needed and the first clue for such an approach comes from Remark 3, namely that the set of poles and the set of zeros of $\chi(x ; \lambda)$ are $\left\{\tau_{n}(0)\right\}_{n=1}^{N}$ and $\left\{\tau_{n}(x)\right\}_{n=1}^{N}$ respectively. 


\section{Extended Abel's Formula}

We start by reminding the reader of the following classical result (see e.g., [29], [45], or [44], Vol. II):

Abel's Theorem. Assume that $\Omega$ is a compact Riemann surface of genus $g$ and $\omega_{1}, \omega_{2}, \ldots, \omega_{g}$ is a basis of the abelian differentials of the first kind (i.e. holomorphic differentials) of $\Omega$. Let $m(\lambda)$ be a meromorphic function on $\Omega$ with poles $p_{1}, p_{2}, \ldots, p_{n}$, and zeros $q_{1}, q_{2}, \ldots, q_{n}$ (counting multiplicities - recall that the number of poles of $m(\lambda)$ must be always equal to the number of its zeros). Then the following formula holds:

$$
\sum_{l=1}^{n} \int_{q_{l}}^{p_{l}} \omega_{j}=\text { a period of } \omega_{j}, \quad j=1,2, \ldots, g
$$

(and, conversely, given $p_{1}, p_{2}, \ldots, p_{n}$, and $q_{1}, q_{2}, \ldots, q_{n}$ on $\Omega$ satisfying the above equations, there is a meromorphic function, which can be constructed with the help of theta functions, having these points as poles and zeros respectively - this is, in fact, the difficult part of the theorem).

Abel's formula is not valid for $\phi(\xi ; \lambda)$ since its Riemann surface $\Gamma$ is not compact (alternatively $\phi(\xi ; \lambda)$ is not meromorphic on $\bar{\Gamma}$ ). However $\phi(\xi ; \lambda)$ has a very specific behavior, as $|\lambda| \rightarrow \infty$ (see (43)), and this enables us to extend Abel's theorem in order to cover this case too. Let us first discuss the Hill case.

5.1. Inverse Hill Theory Revisited. Keeping the notation of Section 4, the holomorphic differentials of $\bar{S}$ are

$$
\theta_{j}=\frac{\lambda^{j-1}}{\sqrt{R(\lambda)}} d \lambda, \quad j=1,2, \ldots, N .
$$

Having in mind that $\left\{\tau_{n}(0)\right\}_{n=1}^{N}$ and $\left\{\tau_{n}(x)\right\}_{n=1}^{N}$ are the poles and the zeros, respectively, of $\chi(x ; \lambda)$ one can write the following extended Abel's formulas:

$$
\sum_{n=1}^{N} \int_{\tau_{n}(0)}^{\tau_{n}(x)} \frac{\lambda^{j-1}}{\sqrt{R(\lambda)}} d \lambda=-2 i \delta_{j N} x+\text { a period of } \theta_{j}, \quad j=1,2, \ldots, N,
$$

where $\delta_{j N}$ is the Kronecker delta. This is already a complete set of evolution equations for the $\tau_{n}(x)$ 's, which in some sense is more preferable than (56) $([\mathbf{4 9}])$. To get a system of differential equations we differentiate with respect to $x$ and have

$$
\sum_{n=1}^{N} \frac{\tau_{n}{ }^{j-1} \tau_{n}^{\prime}}{\sqrt{R\left(\tau_{n}\right)}}=-2 i \delta_{j N}, \quad j=1,2, \ldots, N
$$

Solving for $\tau_{n}^{\prime} / \sqrt{R\left(\tau_{n}\right)}$ (with the help of Vandermonde determinants), we obtain

$$
\frac{\tau_{n}^{\prime}}{\sqrt{R\left(\tau_{n}\right)}}=-\frac{2 i}{\prod_{k \neq n}\left(\tau_{n}-\tau_{k}\right)}, \quad n=1,2, \ldots, N,
$$

which are the evolution equations (56)! 
5.2. Evolutions Equations for the Poles $\mu_{n}(x)$. Let us now discuss the Euler-Bernoulli case (1). We remind the reader that, as a function of $\lambda, \phi(x ; \lambda)$ has $g=2 M+N$ poles $\left\{\mu_{n}(0)\right\}_{n=-2 M, n \neq 0}^{N}, g$ zeros $\left\{\mu_{n}(x)\right\}_{n=-2 M, n \neq 0}^{N}$, and satisfies specific asymptotics, as $|\lambda| \rightarrow \infty$ (see (43)). Let $\omega_{1}, \omega_{2}, \ldots, \omega_{g}$ is a basis of the abelian differentials of the first kind (i.e. holomorphic differentials) of $\bar{\Gamma}$. Then one can write the following extended Abel's formula, which gives the evolution equations for the poles of $\phi(x ; \lambda)$ :

$\sum_{n=-2 M, n \neq 0}^{N} \int_{\mu_{n}(0)}^{\mu_{n}(x)} \omega_{m}=c_{m} \int_{0}^{x}\left[\frac{\rho(y)}{a(y)}\right]^{1 / 4} d y+$ a period of $\omega_{m}, \quad m=1,2, \ldots, g$, where the $c_{m}$ 's are (universal) complex constants.

Differentiating (57) with respect to $x$ yields $g$ first order (nonlinear) differential equations for the $\mu_{n}(x)$ 's.

Remark 6. Since (57) gives $g$ equations, it is essential, as we have already mentioned, that $\phi(x ; \lambda)$ has exactly $g$ poles.

5.3. The Holomorphic Differentials of $\bar{\Gamma}$. In this subsection we describe the holomorphic differentials of $\bar{\Gamma}$. We need $g=2 M+N$ such (linearly independent) differentials. The first $M$ are

$$
\omega_{j}=\frac{\lambda^{j-1}}{\sqrt{P_{E}(\lambda)}} d \lambda, \quad j=1,2, \ldots, M,
$$

where $P_{E}(\lambda)$ is given by $(25)$ (notice that $\operatorname{deg} P_{E}=2 M+1$ ).

The next $M$ differentials are (see (11) and (49))

$$
\omega_{j}=\frac{\lambda^{j-M}}{\sqrt{P_{E}(\lambda)}} \cdot \frac{\Delta^{\prime}(\lambda)}{\sqrt{\Delta(\lambda)^{2}-4}} d \lambda, \quad j=M+1, M+2, \ldots, 2 M .
$$

Remark 7. The differential

$$
\omega_{j}=\frac{1}{\sqrt{P_{E}(\lambda)}} \cdot \frac{\Delta^{\prime}(\lambda)}{\sqrt{\Delta^{2}-4}} d \lambda
$$

is not holomorphic since, as $\lambda \rightarrow 0$,

$$
\frac{1}{\sqrt{P_{E}(\lambda)}} \cdot \frac{\Delta^{\prime}(\lambda)}{\sqrt{\Delta(\lambda)^{2}-4}}=\frac{c}{\lambda^{5 / 4}}+o\left(\frac{1}{\lambda^{5 / 4}}\right)
$$

where $c \neq 0$.

If $N=0,(58)$ and (59) give a complete set of holomorphic differentials. Otherwise we need $N$ more differentials which can be found from the characterization of $\bar{\Gamma}$ given by (54). It is still an open question whether one can find simple closed form expressions for these remaining $N$ differentials, in the case $M>0$ (the case $M=0$ reduces to the second order case - see $[39])$

5.4. The First $M$ Evolution Equations. We start by reminding the reader that $r(\lambda)$ satisfies (5) if and only if $r(\lambda)^{-1}$ satisfies $(5)$. Let $\phi(x ; \lambda)$ be the normalized Floquet solution corresponding to $r(\lambda)$, as defined in Subsection 2.2. We denote by

$$
\phi^{*}(x ; \lambda)
$$

the normalized Floquet solution that corresponds to $r(\lambda)^{-1}$. 
Due to (43) the function

$$
\phi(x ; \lambda) \phi^{*}(x ; \lambda)
$$

is meromorphic on $\bar{\Gamma}$ and remains invariant under the transformation $r(\lambda) \mapsto$ $r(\lambda)^{-1}$. Therefore this function is meromorphic (and, of course, single-valued) on the (compact) Riemann surface of $\sqrt{P_{E}(\lambda)}$ (see (25)). We denote this hyperelliptic surface by $S_{E}$. The genus of $S_{E}$ is $M$ and a basis of holomorphic differentials is

$$
\eta_{j}=\frac{\lambda^{j-1} d \lambda}{\sqrt{P_{E}(\lambda)}}, \quad j=1,2, \ldots, M .
$$

Noting that $\phi(x ; \lambda) \phi^{*}(x ; \lambda)$ has poles $\left\{\mu_{n}(0)\right\}_{n=-2 M, n \neq 0}^{N}$ and zeros $\left\{\mu_{n}(x)\right\}_{n=-2 M, n \neq 0}^{N}$ ( $g=2 M+N$, as before), one can write (the classical) Abel's theorem:

$$
\sum_{n=-2 M, n \neq 0}^{N} \int_{\mu_{n}(0)}^{\mu_{n}(x)} \eta_{j}=\text { a period of } \eta_{j}, \quad j=1,2, \ldots, M .
$$

Again we differentiate with respect to $x$ and get (in view of (60))

$$
\sum_{n=-2 M, n \neq 0}^{N} \frac{\mu_{n}(x)^{j-1}}{\sqrt{P_{E}\left(\mu_{n}(x)\right)}} \mu_{n}^{\prime}(x)=0, \quad j=1,2, \ldots, M .
$$

Of course, given (57) the above equations are redundant.

Using (59) in (57) and differentiating one can write another set of $M$ evolution equations, independent of (61).

5.5. Example: The Case $M=1, N=0$. In this case

$$
g=2 \text {. }
$$

Formula (61) becomes

$$
\frac{\mu_{-1}^{\prime}(x)}{\sqrt{P_{E}\left(\mu_{-1}(x)\right)}}+\frac{\mu_{-2}^{\prime}(x)}{\sqrt{P_{E}\left(\mu_{-2}(x)\right)}}=0 .
$$

To get the second equation we use (59) in (57) which, after differentiating with respect to $x$, yields

(63) $\frac{\mu_{-1} \mu_{-1}^{\prime}}{\sqrt{P_{E}\left(\mu_{-1}\right)}} \cdot \frac{\Delta^{\prime}\left(\mu_{-1}\right)}{\sqrt{\Delta\left(\mu_{-1}\right)^{2}-4}}+\frac{\mu_{-2} \mu_{-2}^{\prime}}{\sqrt{P_{E}\left(\mu_{-2}\right)}} \cdot \frac{\Delta^{\prime}\left(\mu_{-2}\right)}{\sqrt{\Delta\left(\mu_{-2}\right)^{2}-4}}=c_{2}^{\prime}\left[\frac{\rho(x)}{a(x)}\right]^{1 / 4}$,

where $c_{2}^{\prime}$ is a constant.

The formula (62) implies

$$
\frac{d \mu_{-2}}{d \mu_{-1}}=-\frac{\sqrt{P_{E}\left(\mu_{-2}(x)\right)}}{\sqrt{P_{E}\left(\mu_{-1}(x)\right)}},
$$

with

$$
P_{E}(\lambda)=\lambda\left(\lambda-\nu_{1}\right)\left(\lambda-\nu_{2}\right), \quad \nu_{2}<\nu_{1}<0 .
$$

Equation (64) can be solved explicitly. Let us first bring it in the Weierstrassian form, so that we can use the $\wp$-function. We need a minor normalization

$$
u=\mu_{-1}-\nu^{*}, \quad v=\mu_{-2}-\nu^{*}, \quad \text { where } \quad \nu^{*}=\frac{\nu_{1}+\nu_{2}}{3} .
$$


In view of those, (64) becomes

$$
\frac{d u}{\sqrt{\left(u-e_{1}\right)\left(u-e_{2}\right)\left(u-e_{3}\right)}}+\frac{d v}{\sqrt{\left(v-e_{1}\right)\left(v-e_{2}\right)\left(v-e_{3}\right)}}=0,
$$

where

$$
e_{1}=-\nu^{*}, \quad e_{2}=\nu_{1}-\nu^{*}, \quad e_{3}=\nu_{2}-\nu^{*},
$$

so that

$$
e_{1}+e_{2}+e_{3}=0
$$

and hence we can consider the Weierstrass $\wp$-function satisfying

$$
\left(\wp^{\prime}\right)^{2}=4\left(\wp-e_{1}\right)\left(\wp-e_{2}\right)\left(\wp-e_{3}\right) .
$$

It follows that (67), and hence (64), can be integrated as

$$
\wp^{-1}(u)+\wp^{-1}(v)=c,
$$

where $c$ is the constant of integration. Applying $\wp$ to both sides and using the evenness and the addition formula of $\wp$ (see, e.g., $[\mathbf{2 9}]$ ) we get

$$
\left[\frac{\sqrt{\left(v-e_{1}\right)\left(v-e_{2}\right)\left(v-e_{3}\right)}-\sqrt{\left(u-e_{1}\right)\left(u-e_{2}\right)\left(u-e_{3}\right)}}{v-u}\right]^{2}-u-v=\wp(c) .
$$

Therefore (see (66)) one obtains an algebraic equation for the point $\left(\mu_{-1}, \mu_{-2}\right)=$ $\left(u+\nu^{*}, v+\nu^{*}\right)$.

To get a second equation we start with (59) which reduces to

$$
\int_{\mu_{-1}(0)}^{\mu_{-1}(x)} \omega_{2}+\int_{\mu_{-2}(0)}^{\mu_{-2}(x)} \omega_{2}=c_{2} \int_{0}^{x}\left[\frac{\rho(y)}{a(y)}\right]^{1 / 4} d y+\text { a period of } \omega_{2} .
$$

¿From (59) we have

$$
\omega_{2}=\frac{\lambda}{\sqrt{P_{E}(\lambda)}} \cdot \frac{\Delta^{\prime}(\lambda)}{\sqrt{\Delta(\lambda)^{2}-4}} d \lambda .
$$

Thus, substituting $\omega_{2}$ in (69) and differentiating with respect to $x$ yields (70)

$\frac{\mu_{-1}}{\sqrt{P_{E}\left(\mu_{-1}\right)}} \cdot \frac{\Delta^{\prime}\left(\mu_{-1}\right)}{\sqrt{\Delta\left(\mu_{-1}\right)^{2}-4}} \mu_{-1}^{\prime}+\frac{\mu_{-2}}{\sqrt{P_{E}\left(\mu_{-2}\right)}} \cdot \frac{\Delta^{\prime}\left(\mu_{-2}\right)}{\sqrt{\Delta\left(\mu_{-2}\right)^{2}-4}} \mu_{-2}^{\prime}=c_{2}\left[\frac{\rho(x)}{a(x)}\right]^{1 / 4}$,

where $P_{E}(\lambda)$ is given by (65), $\Delta(\lambda)$ is the double-valued function given in (8) (see also (11) and (12)), and $c_{2}$ is a constant associated to the Riemann surface $\bar{\Gamma}$.

Formulas (68) and (70) are the evolution equations for $\mu_{-1}$ and $\mu_{-2}$.

\section{Discussion on the Recovery of $a(x)$ and $\rho(x)$}

Using (20), (23), and (36) one gets

$$
\begin{aligned}
& \frac{E(\lambda) \sqrt{F^{+}(\lambda) F^{-}(\lambda)}}{\lambda^{3 / 2} H(\xi ; \lambda)} \\
& =\frac{i c_{F} c_{E} \prod_{n=1}^{2 M}\left(1-\frac{\lambda}{\nu_{n}}\right) \prod_{n=1}^{\infty}\left(1-\frac{\lambda}{\nu_{n}^{*}}\right)^{2} \sqrt{\prod_{n=1}^{2 N}\left(1-\frac{\lambda}{\lambda_{n}}\right)} \prod_{n=1}^{\infty}\left(1-\frac{\lambda}{\lambda_{n}^{*}}\right)}{c_{H} \prod_{n}\left[1-\frac{\lambda}{\zeta_{n}(\xi)}\right]}
\end{aligned}
$$


where

$$
c_{H}=b^{4}\left(\int_{0}^{b} \frac{d x}{a(x)}\right)^{2} .
$$

For the degenerate gaps $\lambda_{n}^{*}$ and $\psi$-gaps $\nu_{n}^{*}$ we have cancellations (see (37), (38), and (39)) and hence

$$
\frac{E(\lambda) \sqrt{F^{+}(\lambda) F^{-}(\lambda)}}{\lambda^{3 / 2} H(\xi ; \lambda)}=\frac{i c_{F} c_{E} \prod_{n=1}^{2 M}\left(1-\frac{\lambda}{\nu_{n}}\right) \prod_{n=1}^{2 N} \sqrt{\left(1-\frac{\lambda}{\lambda_{n}}\right)}}{c_{H} \prod_{n=-2 M, n \neq 0}^{N}\left[1-\frac{\lambda}{\mu_{n}(\xi)}\right]} .
$$

Letting $|\lambda| \rightarrow \infty$ yields

$$
\lim _{|\lambda| \rightarrow \infty} \frac{E(\lambda) \sqrt{F^{+}(\lambda) F^{-}(\lambda)}}{\lambda^{3 / 2} H(\xi ; \lambda)}=\frac{i c_{F} c_{E}}{c_{H}} \frac{\prod_{n=-2 M, n \neq 0}^{N} \mu_{n}(\xi)}{\prod_{n=1}^{2 M} \nu_{n} \prod_{n=1}^{2 N} \sqrt{\lambda_{n}}} .
$$

On the other hand there is another way to compute the left-hand side limit, namely by using the large $|\lambda|$ asymptotics, which gives

$$
\lim _{|\lambda| \rightarrow \infty} \frac{E(\lambda) \sqrt{F^{+}(\lambda) F^{-}(\lambda)}}{\lambda^{3 / 2} H(\xi ; \lambda)}=16 i a(\xi)^{1 / 2} \rho(\xi)^{3 / 2} .
$$

Therefore

$$
a(\xi)^{1 / 2} \rho(\xi)^{3 / 2}=\frac{c_{F} c_{E}}{16 c_{H}} \frac{\prod_{n} \mu_{n}(\xi)}{\prod_{n=1}^{2 M} \nu_{n} \prod_{n=1}^{2 N} \sqrt{\lambda_{n}}} .
$$

This is an example of a trace formula (since we have products instead of sums, we could call it "determinant formula"). Since we have two unknown functions, $a$ and $\rho$, we need one more equation.

The Baker-Akhiezer function $\phi(x ; \lambda)$ can be written down (via theta functions) with the help of its zeros, i.e. the $\mu_{n}(x)$ 's, its poles, i.e. the $\mu_{n}(0)$ 's, and the differentials of $\bar{\Gamma}$ (see [14]). Substituting this expression of $\phi(x ; \lambda)$ in the original equation (1), we have one more equation involving the $\mu_{n}(x)$ 's and the coefficients $a(x)$ and $\rho(x)$. In fact (1) produces, due to the parameter $\lambda$, an one-parameter family of equations (hence (71) is not needed).

Acknowledgments. The author wishes to express his gratitude to Professors Fritz Gesztesy, Peter Kuchment, Ari Laptev, Henry McKean, Sergey Novikov, Barry Simon, and Stephanos Venakides for helpful discussions and suggested references.

\section{References}

[1] A. Badanin and E. Korotyaev, Quasimomentum of Fourth Order Periodic Operator, preprint, 2001.

[2] V. BARCiLON, Inverse Problem for a Vibrating Beam in the Free-Clamped Configuration, Philosophical Transactions of the Royal Society of London, Series A, 304 (1982), 211-251.

[3] R. Beals and R. R. Coifman, Scattering and Inverse Scattering for First Order Systems, Comm. Pure Appl. Math. 37, no. 1 (1984), 39-90.

[4] R. Beals, P. Deift, and C. Tomei, Direct and Inverse Scattering on the Line, Mathematical Surveys and Monographs, 28. American Mathematical Society, Providence, RI, 1988, xiv +209 pp.

[5] C. M. Bender and S. A. Orszag, "Advanced Mathematical Methods for Scientists and Engineers," McGraw-Hill Book Company, New York, 1978.

[6] R. Carlson, Compactness of Floquet Isospectral Sets for the Matrix Hill's Equation, Proceedings of the American Mathematical Society 128, no. 10 (2000), 2933-2941. 
[7] R. CARlson, Eigenvalue Estimates and Trace Formulas for the Matrix Hill's Equation, Journal of Differential Equations 167, no. 1 (2000), 211-244.

[8] L. F. Caudill, P. A. Perry, and A. W. Schueller, Isospectral Sets for Fourth-Order Ordinary Differential Operators, preprint, 1996.

[9] E. A. Coddington And N. Levinson, "Theory of Ordinary Differential Equations," Robert E. Krieger Publishing Company, Malabar, Florida, 1987.

[10] W. Craig, The Trace Formula for Schrödinger Operators on the Line, Commun. Math. Phys. 126 (1989), 379-407.

[11] M. L. Da Silva Menezes, Infinite genus curves with hyperelliptic ends, Comm. Pure Appl. Math. 42, no. 2 (1989), 185-212.

[12] E. Date and S. Tanaka, Analog of Inverse Scattering Theory for the Discrete Hill's Equation and Exact Solution for the Periodic Toda Lattice, Progress of Theoretical Physics, Vol. 55, No. 2 (February 1976), 457-465.

[13] E. Date and S. Tanaka, Periodic Multi-Soliton Solutions of Korteweg-de Vries Equation and Toda Lattice, Supplement of the Progress of Theoretical Physics, No. 59 (1976), 107-125.

[14] B. A. Dubrovin, I. M. Krichever, And S. P. Novikov, Integrable Systems. I, Dynamical Systems, IV, 177-332, Encyclopaedia of Mathematical Sciences, 4, Springer, Berlin, 2001.

[15] B. A. Dubrovin, V. Matveev, and S. P. Novikov, Nonlinear Equations of KortewegdeVries Type, Finite Zone Linear Operators, and Abelian Varieties, Uspekhi. Mat. Nauk, 31 (1976), 55-136; Russ. Math. Surveys, 31 (1976), 59-146.

[16] N. Dunford and J. T. Schwartz, "Linear Operators. Part II: Spectral Theory; Self Adjoint Operators in Hilbert Space," Wiley Classics Library Edition, New York, 1988.

[17] A. Finkel, E. Isaacson, and E. Trubowitz, An Explicit Solution of the Inverse Periodic Problem Hill's Equation, SIAM J. Math. Anal. 18, No. 1 (Jan. 1987), 46-53.

[18] F. Gesztesy, H. Holden, B. Simon, and Z. Zhao, Trace Formulae and Inverse Spectral Theory for Schrödinger Operators, Bull. Amer. Math. Soc. (New Series) 29 (1993), 250-255.

[19] F. Gesztesy and R. Weikard, Floquet Theory Revisited, in "Differential Equations and Mathematical Physics," Proceedings of the International Conference, Univ. of Alabama at Birmingham, March 13-17, 1994, International Press, 1995.

[20] G. M. L. Gladwell, "Inverse Problems in Vibration," Martinus Nijhoff Publishers, Boston, 1986.

[21] J. Hoppe, A. Laptev, And J. Östensson, Follytons and the Removal of Eigenvalues for Fourth Order Differential Operators, preprint, 2004.

[22] R. Johnson, $m$-Functions and Floquet Exponents for Linear Differential Systems, Annali di Mathematica pura ed applicata (IV), CXLVII (1987), 211-248.

[23] R. Johnson and J. Moser, The Rotation Number for Almost Periodic Potentials, Comm. Math. Phys. 84 (1982), 403-438; erratum: Comm. Math. Phys. 90 (1983), 317-318.

[24] P. Kuchment, "Floquet Theory for Partial Differential Equations," Birkhauser, Verlag, Basel, 1993.

[25] W. Magnus and S. Winkler, "Hill's Equation," Dover Publications, Inc., New York, 1979.

[26] M. M. Malamud, Necessary Conditions for the Existence of a Transformation Operator for Higher Order Equations, Funksional. Anal. i Ego Philozhen. 16 (1982), 74-75. Functional Anal. Appl. 16 (1982), 219-221.

[27] H. P. McKean, Boussinesq's equation on the circle, Comm. Pure Appl. Math. 34, no. 5 (1981), 599-691.

[28] H. P. McKean, private communication (April 2003).

[29] H. P. McKean and V. Moll, "Elliptic Curves; Function Theory, Geometry, Arithmetic," Cambridge University Press, New York, 1999.

[30] H. P. McKean and E. Trubowitz, Hill's Operator and Hyperelliptic Function Theory in the Presence of Infinitely Many Branch Points, Comm. Pure Appl. Math. 29, no. 2 (1976), 143-226.

[31] H. P. McKean and P. van Moerbeke, The Spectrum of Hill's Equation, Invent. Math. 30, no. 3 (1975), 217-274.

[32] J. R. McLaughlin, On Constructing Solutions to an Inverse Euler-Bernoulli Problem, in "Inverse Problems of Acoustic and Elastic Waves," pp341-347, F. Santosa, et al. (editors), Philadelphia: SIAM, 1984.

[33] J. R. McLaughlin, Analytical Methods for Recovering Coefficients in Differential Equations from Spectral Data, SIAM Review 28 (1986), 53-72. 
[34] R. E. Miller, The Eigenvalue Problem for a Class of Long, Thin Elastic Structures with Periodic Geometry, Quarterly of Applied Mathematics, LII, No. 2 (June 1994), 261-282.

[35] M. A. Naimark, "Linear Differential Operators," Part I \& II, Frederick Ungar Publishing Co., New York, 1967 \& 1968

[36] S. P. Novikov, private communication (April 2001).

[37] V. G. Papanicolaou, The Spectral Theory of the Vibrating Periodic Beam, Comm. Math. Phys. 170 (1995), 359-373.

[38] V. G. Papanicolaou, The Periodic Euler-Bernoulli Equation, Transactions of the American Mathematical Society 355, No. 9 (2003), 3727-3759.

[39] V. G. Papanicolaou, An Inverse Spectral Result for the Periodic Euler-Bernoulli Equation, Indiana University Mathematics Journal 53, No. 1 (2004), 223-242

[40] V. G. Papanicolaou and D. Kravvaritis, An Inverse Spectral Problem for the EulerBernoulli Equation for the Vibrating Beam, Inverse Problems 13 (1997), 1083-1092.

[41] V. G. Papanicolaou and D. Kravvaritis, The Floquet Theory of the Periodic EulerBernoulli Equation, Journal of Differential Equations 150 (1998), 24-41.

[42] M. Reed and B. Simon, "Methods of Modern Mathematical Physics, VI: Analysis of Operators," Academic Press, New York, 1978.

[43] L. A. Sachnovich, Inverse Problems for Differential Equations of Order $n>2$ with Analytic Coefficients, Matematicheskii Sbornik 46 (1958), 61-76.

[44] C. L. Siegel, "Topics in Complex Function Theory," 3 volumes, Wiley, New York, N. Y., 1969, 1971, 1973.

[45] G. Springer, "Introduction to Riemann Surfaces," Chelsea Publishing Company, New York, N. Y., 1981.

[46] S. Timoshenko And D. H. Young, "Elements of Strength of Materials," 5th Edition, D. Van Nostrand Company, Inc., Princeton, NJ, 1968.

[47] E. C. Titchmarsh, "The Theory of Functions," Second Edition, Oxford University Press, 1939.

[48] E. Trubowitz, The Inverse Problem for Periodic Potentials, Comm. Pure Appl. Math. 30, no. 3 (1977), 321-337.

[49] S. VENAKIDES, private communication.

Department of Mathematics, National Technical University of Athens, Zografou Campus, 157 80, Athens, GREECE

E-mail address: papanico@math.ntua.gr 